\title{
Adult age differences in refreshing and elaboration and their consequences for working memory and long-term memory
}

\author{
Lea M. Bartsch and Klaus Oberauer \\ University of Zurich and University Priority Program Dynamics of Healthy Aging
}

Author Note

We thank Lina Lane and Sabrina Trachsler for helping with data collection. The behavioral data and corresponding analysis scripts can be accessed in the Open Science Framework (https://osf.io/h2er9). Correspondence should be addressed to Lea M. Bartsch, Department of Psychology, Cognitive Psychology Unit, University of Zurich, Binzmuehlestrasse 14/22, 8050 Zurich, Switzerland. E-mail: 1.bartsch@psychologie.uzh.ch. Tel: +41 446357461 


\begin{abstract}
Older adults show a pronounced decline in long-term memory (LTM), but the source of this deficit is still debated. The present study investigated whether deficient engagement in refreshing and elaboration at the level of working memory (WM) causes this deficit. Our results show that the benefit of refreshing in WM was unaffected by age. Refreshing had no effect on LTM in both young and older adults. Elaboration benefited LTM in young adults, but not in older adults. Therefore, the LTM deficit of older adults might arise at least in part from a deficit in the process of elaboration.
\end{abstract}

Keywords: Refreshing, Elaboration, Working memory, Long-term memory, Aging 


\section{Introduction}

Aging has been repeatedly associated with declines in episodic long-term memory (LTM), and it has been proposed that the underlying cause lies in a decline in working memory (WM) maintenance processes in older age (Hoareau, Lemaire, Portrat, \& Plancher, 2016; Plancher, Boyer, Lemaire, \& Portrat, 2017; Smith, 1980). Such maintenance processes differ in the extent to which they are assumed to change the quality of representations they act upon. Therefore, they could differentially influence the age-related decline in memory.

Two of these processes are currently the focus of discussion: elaboration and refreshing. Initially, it has been proposed that by semantically enriching representations in WM through elaboration, these transform into associative structures in LTM, and thus benefit WM in two ways: 1) Information transferred into LTM frees capacity in WM and 2) elaborately enriched representations provide stronger retrieval cues than those of representations that were simply repeated through articulatory rehearsal. Further, a maintenance process called attentional refreshing has been proposed, which has been shown to be distinct from articulatory rehearsal and elaboration (Bartsch, Singmann, \& Oberauer, 2018; Camos et al., 2018; Johnson, Reeder, Raye, \& Mitchell, 2002; Raye, Johnson, Mitchell, Greene, \& Johnson, 2005). Refreshing is understood as a domain-general process through which representations in WM are reactivated during maintenance. It is yet unclear what mechanism underlies its potential benefit for memory, with some researchers arguing that refreshing strengthens the bindings between items and their contexts, thereby creating stronger retrieval-cues for WM as well as LTM (Barrouillet \& Camos, 2012; Johnson et al., 2002; Souza, Rerko, \& Oberauer, 2015).

In the present study, we argue that if representations in WM can be strengthened through processes such as elaboration or refreshing, this would become evident in WM as well as LTM 
performance. Moreover, any deficiency related to the processing (formation, maintenance, strengthening) of representations in WM in old age should contribute to both WM deficits and LTM deficits in older adults.

\section{Effects of Age on Elaboration}

A previous study has provided first evidence that the direct manipulation of elaboration on the level of WM had no benefit for older adults' LTM compared to repeated reading, which stands in direct contrast to the benefit showed by young adults (Bartsch, Loaiza, Jäncke, Oberauer, \& Lewis-Peacock, 2019). These findings were supported further by fMRI data: Both young and older adults showed differentiable brain activation patterns during elaboration and repeated reading, but the degree of neural separability of these two processes was correlated to LTM performance only in young adults.

We conclude from those findings that older adults might be less likely than younger adults to benefit from elaboration, thereby resulting in deficient retention. Early work has supported this claim - referred to as the elaboration deficit hypothesis (Smith, 1980) - showing that older adults are able to capitalize on experiment-administered elaborative strategies but show deficiencies in generating elaborative strategies themselves (Rankin \& Collins, 1985, see also Kamp \& Zimmer, 2015). Taken together, the LTM deficit of older adults might arise at least in part from a deficit in the process of elaboration.

\section{Effects of Age on Refreshing}

Research on the process of refreshing has continuously focused on age-related differences in its effect on memory. Early work provided evidence for an age-related slowing of the process 
as well as a deficit of older adults in deriving a long-term memory benefit from refreshing verbal information compared to younger adults, which was further associated with reduced refreshingrelated brain activity in the left dlPFC (Johnson, Mitchell, Raye, \& Greene, 2004; Raye, Mitchell, Reeder, Greene, \& Johnson, 2008).

Using the retro-cue paradigm to guide attention in WM, three recent studies (Loaiza \& Souza, 2018, 2019; Souza, 2016) have shown that older adults are well able to use single and multiple cues to sequentially refresh visual WM representations, thereby supporting the view of a preserved refreshing benefit for visual WM with age. Nevertheless, they also provided evidence that older adults show a deficit in shielding this benefit of refreshing against distraction.

A previous study investigated the effect of refreshing on WM as well as LTM for verbal material in older adults while measuring process related brain activation patterns with fMRI. Like younger adults, older adults showed a benefit of refreshing compared to unrefreshed items of the same trial in WM (Bartsch et al., 2019). However, this study found no beneficial effects of refreshing compared to repeated reading in LTM, not even in young adults, contrary to previous research (Johnson et al., 2004; Raye et al., 2008).

\section{The present study}

In the present study we aim to investigate whether any age-related deficit in refreshing or elaboration could be a limiting factor for memory performance and may explain age variations in WM as well as LTM. We aim at directly replicating the behavioral results of a previous study (Bartsch et al., 2019). We predict to replicate the findings from that study: Both young and old adults can prioritize a subset of items in WM through refreshing, but this has no impact on their subsequent episodic LTM for these items, so that refreshing is unlikely to be responsible for age 
differences in LTM. In contrast, elaboration resulted in better episodic LTM in young but not old adults, Therefore, the deficiency in long-term memory of older adults might at least in part derive from a deficient engagement in the process of elaboration.

\section{Method}

\section{Participants}

We recruited 28 healthy, right-handed young adults $(23$ females; mean age $=23.75, \mathrm{SD}=$ 3.3 years) from the student population of the University of Zurich as well as 24 healthy, righthanded older adults from the community (14 females; mean age $=69.61, \mathrm{SD}=2.81$ years). The study was approved by the ethical review board of the canton of Zurich. The participants were compensated with either 15 Swiss Francs (about 15 USD) or partial course credit for the onehour session. Cognitive functioning was screened with the Mini-Mental Status Examination (MMSE; Folstein, Folstein, \& McHugh, 1975), indicating age-typical cognitive abilities in our sample of older adults $(\mathrm{M}=28.96, \mathrm{SD}=1.49$, range $=25-30)$. Table 1 of the supplementary material shows the descriptive statistics and posterior distributions of the age effects of our sample. The evidence indicates fewer years of formal education in the older compared with the young adults. The older adults showed better performance than the young adults in a computerized vocabulary test (MWT-B; Mehrfachwahl-Wortschatz Test Version B, Lehrl, 2005), consisting of 37 items in which participants are supposed to find an existing word between four similarly sounding non-words. The MWT-B is a marker test for crystallized intelligence. Furthermore, we assessed processing speed using the digit-symbol task, a subtest of the WAIS (Petermann \& Wechsler, 2012), and the younger adults showed better performance than the older 
adults in this cognitive marker as well. Hence, our sample of young and old adults show typical differences in education and measures of crystallized intelligence (Li et al., 2004).

\section{Paradigm}

The paradigm is the same as reported in two recent studies (Bartsch et al., 2019, 2018). We asked participants to remember six nouns in serial order (see Error! Reference source not found.). After list presentation, either the first three words or the last three words were to be processed again in one of four ways, depending on the experimental condition. During encoding it was not predictable which half of the items would have to be processed. In the repeat condition, the three words appeared again sequentially on the screen, and the subjects had to simply re-read them silently. In the refreshing condition, the to-be-processed words were replaced by refreshing prompts appearing at the same location. The subjects were instructed to "think of" the corresponding words as soon as the prompts were shown. In the elaboration condition, the three to-be-processed words were shown again sequentially on the screen, and subjects were instructed to generate a vivid mental image of the three objects interacting. The stimuli appearing on the screen in that condition did not differ from the repeat condition, leaving the instruction to form a vivid mental image as the only difference between these conditions. Finally, in the combined refreshing with elaboration condition the participants had to "think of" the words replaced by the prompts, and additionally form a vivid mental image of those items. Again, the event sequence of this condition does not differ from the refreshing condition apart from the instruction to form a mental image. The experiment used a 2 × 2 × 2 × 2 (repeat/refresh [repeat, refresh] x elaboration [with elaboration, without elaboration] x processing [processed triplet, unprocessed triplet] x age [young adults, older adults]) within-subject, between-age group design. 


\section{Procedure: Working Memory task}

Participants' memory for each list item was tested in their order of presentation using a 4alternative forced-choice procedure. All test sets included the target (i.e., correct) word, one lure from the same triplet of words within the present list, one lure from the other triplet of the present list, and one new word. This choice had to be made for each of the serial positions successively with a button press. We applied this 4-alternative forced-choice recognition task in order to test both memory for items (i.e., discriminating between items that have been presented in the current memory list and new items) and for serial order (i.e., discriminating between the item in the tested position and other list items). Within each block of four trials, the same type of processing was instructed throughout, and a screen repeating the instructions of the particular condition was shown prior to the beginning of each block. The order of the condition blocks was randomized between subjects.

\section{Procedure: Long-Term Memory Task}

At the end of the last WM block, subjects performed a two minutes' distractor task, in which they judged the correctness of multiplication equations (e.g. $5 \times 9=40$ ?) before the beginning of the LTM task. During this LTM task we assessed participants' LTM for the words they had encoded for the WM tests throughout the experiment. To this end, we presented in each trial the first word of a triplet from one of the studied memory lists. We asked participants to choose, again from four different options, the word that had followed the target word in that triplet. The probe words included the correct word (i.e., which could be either the word in the second or third position of the target triplet for the first prompt, and the fifth or sixth word for the second prompt), two words from another list, and a new word. This allowed us to keep the format of the LTM test very similar to the WM test, and furthermore to compare in each trial the 
memory performance for words from the processed and from the not-processed triplets. As in the WM test, the LTM test also provided information about both item memory (i.e., which words have been presented in the experiment) and relational memory (i.e., which words have been together in a triplet). The participants were made aware of the LTM test before the start of the experiment.

\section{Materials}

The stimuli were nouns randomly drawn from a pool of 863 German abstract and concrete nouns for each subject. The nouns were between three and 15 letters long and had a mean normalized lemma frequency of 30.81/million (drawn from the dlexdb.de lexical database).

\section{Data Analysis}

All data and analysis scripts can be accessed on the Open Science Framework (https://osf.io/h2er9). We analyzed the data using a Bayesian generalized linear mixed model (BGLMM) implemented in the R package rstanarm (Stan Development Team, 2018) following the exact analysis pipeline reported by Bartsch and colleagues $(2018,2019)$. The dependent variable was the number of correct and incorrect responses in each cell of the design per participant. Correct responses were defined as choosing the target item from the four alternatives. Bayesian procedures provide posterior probability distributions of the model parameters (i.e., the regression weights) that express uncertainty about the estimated parameters. The highest density regions (HDRs) of these posteriors can be used for statistical inference. A 95\% HDR represents the range in which the true value of a parameter lies with probability 0.95 , given model and data (Morey, Hoekstra, Rouder, Lee, \& Wagenmakers, 2016). If zero lies outside the Bayesian HDR there is strong evidence for the existence of the corresponding effect. Although the strength of 
evidence varies continuously, for simplicity we will describe effects as "credible" if their HDRs exclude zero. We used an MCMC algorithm (implemented in Stan; Carpenter et al., 2017) that estimated the posteriors by sampling parameter values proportional to the product of prior and likelihood. These samples are generated through 4 independent Markov chains, with 1000 warmup samples each, followed by 1000 samples drawn from the posterior distribution which were retained for analysis. Following Gelman and colleagues (2013), we confirmed that the 4 chains converged to the same posterior distribution by verifying that the $\hat{R}$ statistic - reflecting the ratio of between-chain variance to within-chain variance - was $<1.02$ for all parameters, and we visually inspected the chains for convergence.

\section{Results}

We replicated the majority of the effects of the young and older adults reported in previous studies (Bartsch et al., 2019, 2018). Figure 2 shows the estimated proportion of correct responses and their corresponding 95\% highest posterior density regions for the immediate and delayed memory data. The posterior effect estimates are presented in Table 1. A first question was whether our manipulation of processing half of a memory list had an effect on memory. The credible main effect of processing on immediate memory supported an effect of our manipulation: Participants performed better in the WM test for items that were processed again after initial encoding than for items from the unprocessed triplets (see Table 1 and Figure 2Error! Reference source not found.). There was also a main effect of age, such that older adults showed worse memory performance on tests of both WM and LTM. 


\section{Working memory performance}

We first tested how the effect of refreshing a subset of words in WM compares to the effect of repeated reading of these words. This is the comparison through which Johnson and colleagues evaluated the effect of refreshing on delayed memory (Johnson et al., 2002; Raye et al., 2007). There was a main effect of repeat/refresh (Table 1), but with an advantage of repeating over refreshing. This main effect was further qualified by the two-way interaction of processing and repeat/refresh, indicating that repeated words benefited more from being processed again than refreshed words did. Nevertheless, a beneficial effect of processing was found for both repeated words $(\Delta=0.16,95 \% \operatorname{HDR}=[0.13,0.18])$ and refreshed words $(\Delta=0.06,95 \% \mathrm{HDR}=$ $[0.03,0.09])$. There was no credible evidence for an interaction of age with the effect of processing or repeat/refresh, indicating that older adults benefited similarly to young adults from both processing as well as repeating over refreshing.

The BGLMM revealed no credible evidence for a main effect of elaboration on WM performance, or for any of the interactions involving elaboration (see Table 1).

\section{Long-term memory performance}

The BGLMM revealed evidence for a main effect of elaboration on LTM performance, with better performance in trials with elaboration than without (see Table 1). This main effect was further qualified by an interaction with the repeat/refresh factor indicating that the elaboration benefit was only present for repeated $(\Delta=0.08,95 \% \mathrm{HDR}=[0.04,0.12])$ and not for refreshed trials $(\Delta=-0.01,95 \% \operatorname{HDR}=[-0.04,0.03])$.

There was no evidence for any further interaction including the repeat/refresh factor.

Hence, contrary to the findings of Johnson and colleagues, refreshing did not lead to better LTM than repeated reading. Note that the above pattern of results also holds for a lenient score of 
performance in the LTM task, counting all responses showing correct item memory (i.e. the target, same-list items, and other-list items) as correct responses.

Furthermore, the analysis of the LTM data revealed evidence for an interaction of elaboration with age (see Table 1). Follow-up analyses of the interaction revealed that a beneficial effect of elaboration appeared only for young $(\Delta=0.07,95 \% \operatorname{HDR}=[0.03,0.12])$, but not older adults $(\Delta=0,95 \% \mathrm{HDR}=[-0.04,0.04])$. In sum, memory was better for trials with instructed elaboration than for those without, but only for the young and not the older adults. The above evidence speaks for an age-dependent beneficial effect of elaboration on LTM that is lost in older age.

To summarize, our results provide no evidence for an effect of refreshing on LTM for either age group; instead we replicated the benefit of elaboration on LTM but only for young adults.

\section{Discussion}

The goal of the present study was to investigate whether any deficit in the engagement of WM processes explains any LTM deficits of older adults. To this end we directly manipulated two candidate processes - namely refreshing and elaboration - in a verbal WM task and found differential effects of both processes across age: Refreshing affected neither young nor older adults' LTM. Elaboration improved episodic LTM of young but not old adults. We discuss these findings in the following. 


\section{Impacts of refreshing on memory are preserved in older adults}

The analyses of the immediate memory data revealed that older adults similarly benefited from processing the items again in the refreshing condition, compared to the young adults, as shown in evidence against an interaction of age with the repeat/refresh by processing interaction (see Table 1). Hence, both age groups were equally effective in prioritizing the refreshing items over to the not-processed items in WM. These findings are a direct replication of a previous study, lending further support for the robustness of the effect (Bartsch et al., 2019). Further, the present results add to previous findings of a preserved ability to prioritize items in visual WM in older adults (Gilchrist, Duarte, \& Verhaeghen, 2016; Loaiza \& Souza, 2018; Souza, 2016) and extends them to the verbal domain.

As for the young adults, refreshing had no benefit on LTM in older adults. Although this replicates the age-group specific findings of Johnson (2004), this does not explain the age-related LTM deficit, as refreshing was not related to LTM performance of young adults either. A key difference between our study and that of Johnson (2004) is that in Johnson's study participants had to refresh single items, compared to three items here. Nevertheless, we argue that participants were well able to capitalize on refreshing, given the processing benefit in WM. Therefore a higher memory load cannot explain the lack of a refreshing benefit in LTM. Taken together, we conclude that deficits in refreshing are not responsible for the LTM deficit in older adults.

\section{Elaboration is a contributing factor to age-related deficits in LTM}

Elaboration applied to the memoranda at the stage of WM maintenance had no immediate effect, as shown by the analysis of the immediate memory task, replicating recent work (Bartsch 
et al., 2018). Most importantly to the goal of the study, there was no LTM benefit of elaboration in older adults, whereas this effect was robust in the young group. The LTM deficit in older adults can be attributed at least in part to deficient engagement in the process of elaboration.

One could argue that, as there is neither a benefit of forming a mental image (the elaboration instruction here) in WM nor in LTM for the older adults, they did not engage in the elaboration conditions any differently than they did in the repeat or refreshing condition. Although we cannot directly rule out this alternative explanation with the dataset at hand, the fMRI data of our previous study using this very paradigm provided evidence of differential brain activation patterns for all four processing conditions, meaning that older adults did in fact engage in a different process when instructed to elaborate than when instructed to refresh.

One question that remains is why older adults fail to benefit from elaboration - are they unable to generate enriched memory representations that support episodic memory, or do they not profit from enriched memory representations? Future research could answer this question by providing older adults with the elaborative content (e.g. with images or sentences) to investigate whether this reduces their the LTM deficit. Taken together, our results provide evidence that the LTM deficit of older adults might arise at least in part from a deficit in the process of elaboration. 


\section{References}

Barrouillet, P., \& Camos, V. (2012). As Time Goes By: Temporal Constraints in Working Memory. Current Directions in Psychological Science, 21(6), 413-419. https://doi.org/10.1177/0963721412459513

Bartsch, L. M., Loaiza, V. M., Jäncke, L., Oberauer, K., \& Lewis-Peacock, J. A. (2019). Dissociating refreshing and elaboration and their impacts on memory. BioRxiv, 423277. https://doi.org/10.1101/423277

Bartsch, L. M., Singmann, H., \& Oberauer, K. (2018). The effects of refreshing and elaboration on working memory performance, and their contributions to long-term memory formation. Memory \& Cognition. https://doi.org/10.3758/s13421-018-0805-9

Camos, V., Johnson, M. R., Loaiza, V. M., Portrat, S., Souza, A. S., \& Vergauwe, E. (2018). What is attentional refreshing in working memory? Annals of the New York Academy of Sciences, 1-14. https://doi.org/10.1111/nyas.13616

Carpenter, B., Gelman, A., Hoffman, M. D., Lee, D., Goodrich, B., Betancourt, M., ... Riddell, A. (2017). Stan: A Probabilistic Programming Language. Journal of Statistical Software, 76(1). https://doi.org/10.18637/jss.v076.i01

Gelman, A., Carlin, J. B., Stern, H. S., Dunson, D. B., Vehtari, A., \& Rubin, D. B. (2013). Bayesian data analysis, 3rd edition. Chapman \& Hall/CRC.

Gilchrist, A. L., Duarte, A., \& Verhaeghen, P. (2016). Retrospective cues based on object features improve visual working memory performance in older adults. Neuropsychology, Development, and Cognition. Section B, Aging, Neuropsychology and Cognition, 23(2), 184-195. https://doi.org/10.1080/13825585.2015.1069253

Hoareau, V., Lemaire, B., Portrat, S., \& Plancher, G. (2016). Reconciling Two Computational 
Models of Working Memory in Aging. Topics in Cognitive Science, 8(1), 264-278. https://doi.org/10.1111/tops.12184

Johnson, M. K., Mitchell, K. J., Raye, C. L., \& Greene, E. J. (2004). An age-related deficit in prefrontal cortical function associated with refreshing information. Psychological Science, 15(2), 127-132. https://doi.org/10.1016/j.neuroscience.2008.09.014

Johnson, M. K., Reeder, J. a, Raye, C. L., \& Mitchell, K. J. (2002). Second thoughts versus second looks: an age-related deficit in reflectively refreshing just-activated information. Psychological Science : A Journal of the American Psychological Society / APS, 13(1), 6467. https://doi.org/10.1111/1467-9280.00411

Lehrl, S. (2005). Mehrfachwahl-Wortschatz-Intelligenztest MWT-B (5th ed.). Balingen: Spitta Verlag.

Li, S.-C., Lindenberger, U., Hommel, B., Aschersleben, G., Prinz, W., \& Baltes, P. B. (2004). Transformations in the couplings among intellectual cognitive processes across the life span. Psychological Science, 15(3), 155-163. https://doi.org/10.1111/j.09567976.2004.01503003.x

Loaiza, V. M., \& Souza, A. S. (2018). Is refreshing in working memory impaired in older age ? Evidence from the retro-cue paradigm. Annals of the New York Academy of Sciences, (Special Issue: Attention in Working Memory), 13-15. https://doi.org/10.1111/nyas.13623

Loaiza, V. M., \& Souza, A. S. (2019). An age-related deficit in preserving the benefits of attention in working memory. Psychology and Aging. https://doi.org/10.1037/pag0000326

Morey, R. D., Hoekstra, R., Rouder, J. N., Lee, M. D., \& Wagenmakers, E.-J. (2016). The fallacy of placing confidence in confidence intervals. Psychonomic Bulletin \& Review, 23(1), 103123. https://doi.org/10.3758/s13423-015-0947-8 
Petermann, F., \& Wechsler, D. (2012). Wechsler Adult Intelligence Scale - Fourth Edition (WAIS$I V)$. Frankfurt am Main: Pearson.

Plancher, G., Boyer, H., Lemaire, B., \& Portrat, S. (2017). Under Which Conditions Can Older Participants Maintain Information In Working Memory? Experimental Aging Research, 43(5), 409-429. https://doi.org/10.1080/0361073X.2017.1369730

Rankin, J. L., \& Collins, M. (1985). Adult age differences in memory elaboration. Journal of Gerontology, 40(4), 451-458.

Raye, C. L., Johnson, M. K., Mitchell, K. J., Greene, E. J., \& Johnson, M. R. (2005). Refreshing: A Minimal Executive Function. Control, 43(1), 135-145. https://doi.org/10.1016/S0010$9452(08) 70451-9$

Raye, C. L., Mitchell, K. J., Reeder, J. a, Greene, E. J., \& Johnson, M. K. (2008). Refreshing one of several active representations: behavioral and functional magnetic resonance imaging differences between young and older adults. Journal of Cognitive Neuroscience, 20(5), 852862. https://doi.org/10.1162/jocn.2008.20508

Smith, A. D. (1980). Age differences in encoding, storage, and retrieval. New Directions in Memory and Aging, 23-46.

Souza, A. S. (2016). No age deficits in the ability to use attention to improve visual working memory. Psychology and Aging, 31(5), 456-470. https://doi.org/10.1037/pag0000107

Souza, A. S., Rerko, L., \& Oberauer, K. (2015). Refreshing memory traces: thinking of an item improves retrieval from visual working memory. Annals of the New York Academy of Sciences, 1339(1), 20-31. https://doi.org/10.1111/nyas.12603

Stan Development Team. (2018). rstanarm: $\{$ Bayesian $\}$ applied regression modeling via $\{$ Stan $\}$. Retrieved from http://mc-stan.org/ 
Tables

Table 1 The posterior effect estimates and their 95\% HDRs of the generalized linear mixed model for binomial response variables for the immediate serial and delayed memory data.

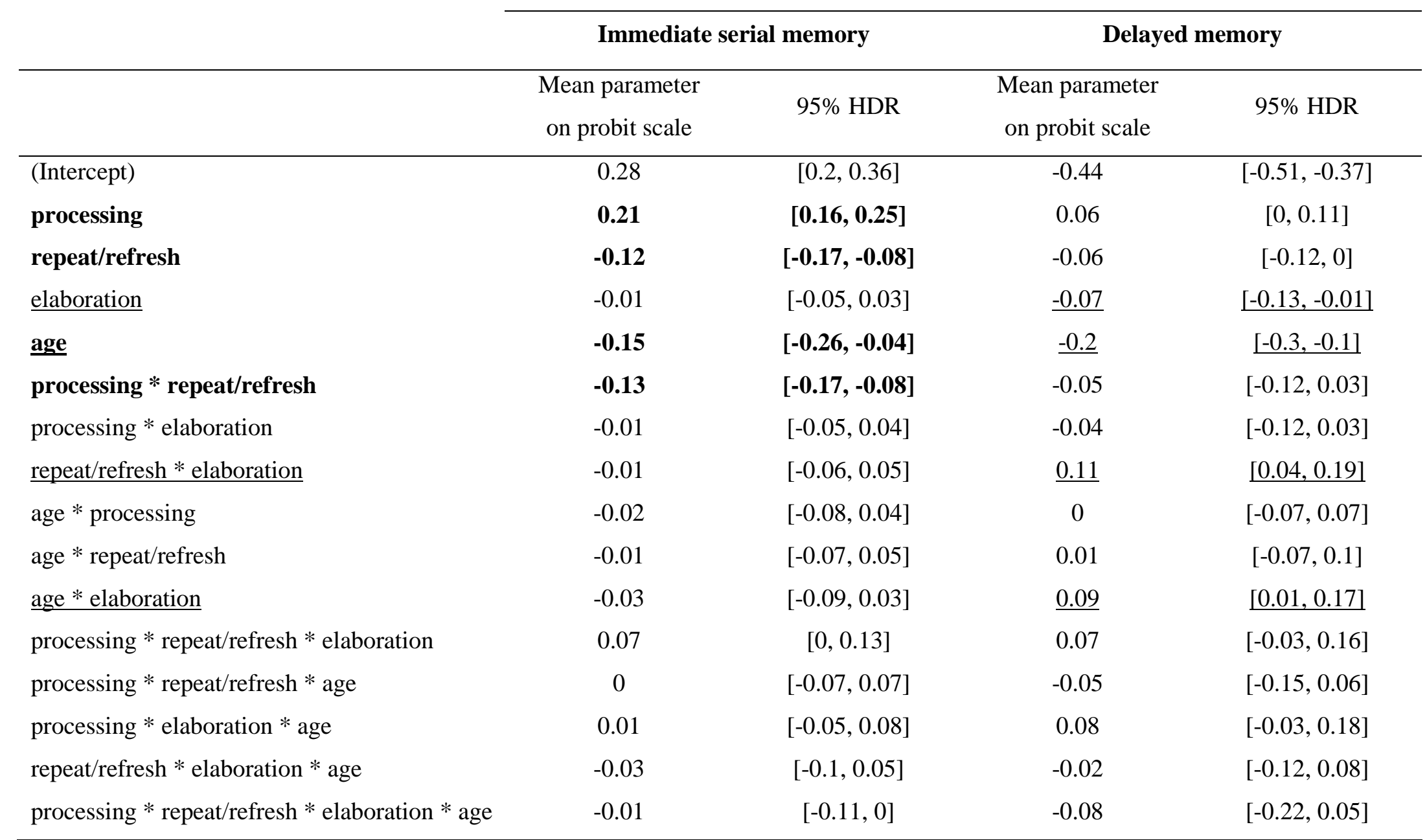

Note. Credible effects, defined as HDRs excluding zero, are printed in bold (immediate memory) and are underlined (delayed memory). 
Figures



Figure 1 Illustration of the working memory paradigm of Experiment 1. Subjects were shown a list of six words sequentially, followed by either the first or second triplet being processed according to the four experimental conditions. 

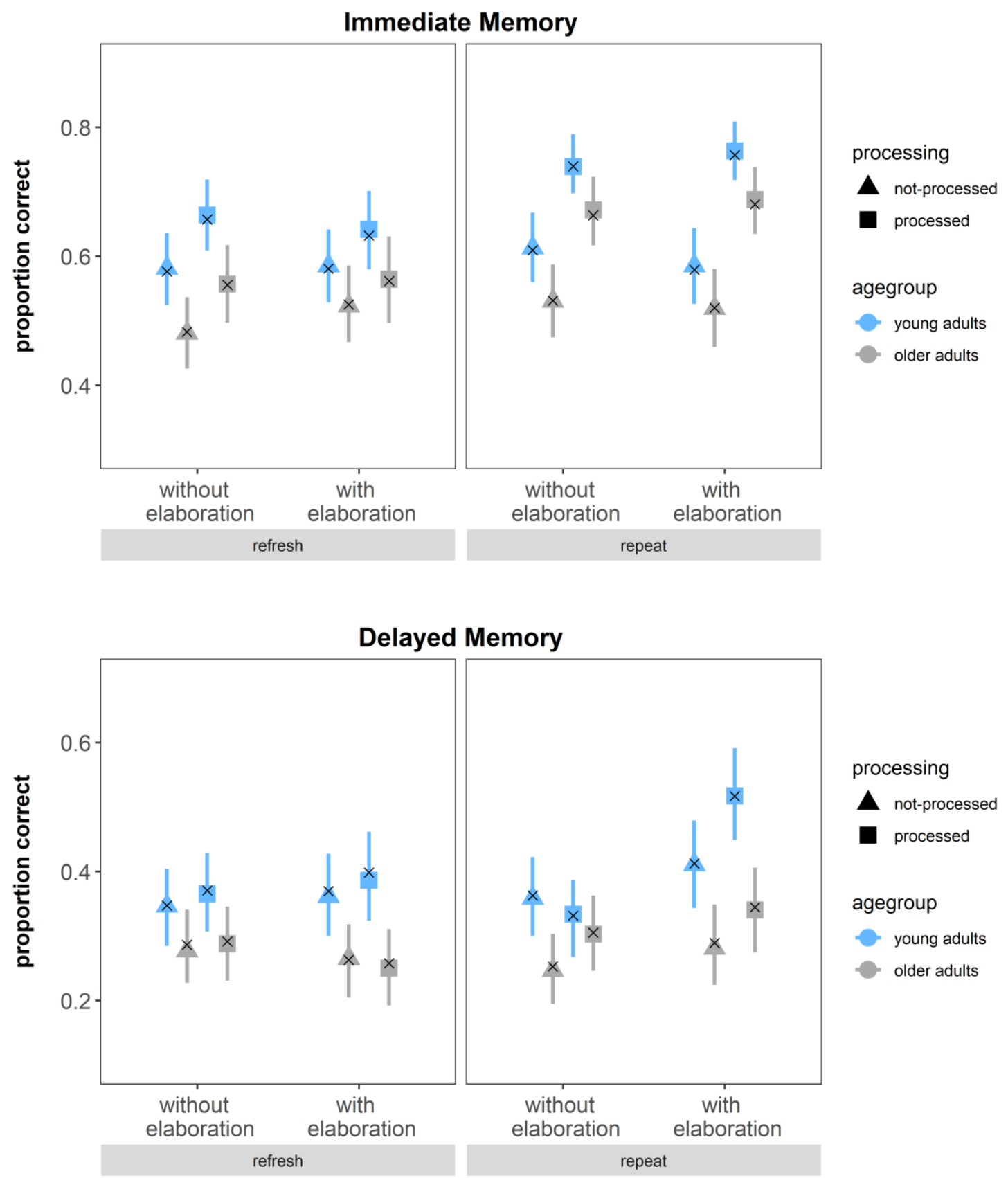

Figure 2 Proportion correct in the immediate (WM) and delayed (LTM) memory task. The grey symbols and error bars represent estimated proportions and their 95\% HDRs from the BGLMM for young adults and the blue symbols those of the older adults. The crosses represent the observed proportions. Their overlap indicates that the model adequately describes the data. 